\title{
Understanding preservation theorems, II
}

\author{
Chaz Schlindwein \\ Department of Mathematics and Computing \\ Lander University \\ Greenwood, South Carolina 29649 USA \\ cschlind@lander.edu
}

November 18, 2018

\begin{abstract}
We present an exposition of much of Sections VI.3 and XVIII.3 from Shelah's book Proper and Improper Forcing. This covers numerous preservation theorems for countable support iterations of proper forcing, including preservation of the property "no new random reals over $V$," the property "reals of the ground model form a non-meager set," the property "every dense open set contains a dense open set of the ground model," and preservation theorems related to the weak bounding property, the weak ${ }^{\omega} \omega$-bounding property, and the property "the set of reals of the ground model has positive outer measure."
\end{abstract}




\section{Introduction}

This is the fourth of a sequence of papers giving an exposition of portions of Shelah's book, Proper and Improper Forcing [9]. The earlier papers were [6], [7], and [8], which cover sections 2 through 8 of [9, Chapter XI], sections 2 and 3 of [9, Chapter XV], and sections 1 and 2 of [9, Chapter VI], respectively.

In this paper, we give an exposition of much of [9, Sections VI.3 and XVIII.3] dealing with preservation theorems. We include proofs of the preservation, under countable support iteration of proper forcing, of the property "no new random reals," the property "every open dense set contains an old open dense set," the property of non-meagerness of the reals of the ground model, and preservation theorems related to weak bounding, weak ${ }^{\omega} \omega$-bounding, and "the set of reals of the ground model has positive outer measure."

Another treatment of preservation theorems, using different methods, is given in [2], [3]. The results of [9, Section VI.3] included here as Theorem 2.5, Theorem 3.5, and Theorem 4.13 may also be derived as corollaries of [1, Theorem 6.1.18]; the proof there is essentially the same as the ones given by Shelah in $[9$, Section VI.3].

\section{Preservation of weak bounding}

The most important tool in the study of preservation theorems for countable support forcing iterations is the Proper Iteration Lemma. Here, and throughout this paper, $P_{\alpha, \kappa}$ is characterized by

$$
V\left[G_{P_{\alpha}}\right] \models " P_{\alpha, \kappa}=\left\{p \uparrow[\alpha, \kappa): p \in P_{\kappa} \text { and } p \uparrow \alpha \in G_{P_{\alpha}}\right\} . "
$$

Theorem 2.1 (Proper Iteration Lemma, Shelah). Suppose $\left\langle P_{\eta}: \eta \leq \kappa\right\rangle$ is a countable support forcing iteration based on $\left\langle Q_{\eta}: \eta<\kappa\right\rangle$ and for every $\eta<\kappa$ we have that $\mathbf{1} \|_{P_{\eta}}$ " $Q_{\eta}$ is proper." Suppose also that $\alpha<\kappa$ and $\lambda$ is a sufficiently large regular cardinal and $N$ is a countable elementary submodel of $H_{\lambda}$ and $\left\{P_{\kappa}, \alpha\right\} \in N$ and $p \in P_{\alpha}$ is $N$-generic and $p \Vdash$ " $q \in P_{\alpha, \kappa} \cap N\left[G_{P_{\alpha}}\right]$." Then there is $r \in P_{\kappa}$ such that $r$ is $N$-generic and $r \uparrow \alpha=p$ and $p \Vdash$ " $r \uparrow[\alpha, \kappa) \leq q$."

Proof: See (e.g.) [8, Theorem 2.1].

We deal first with the weak bounding property. 
Definition 2.2. Suppose $A$ and $B$ are sets of integers. We say $A \subseteq^{*} B$ iff $\{n \in A: n \notin B\}$ is finite.

Definition 2.3. Suppose $\mathcal{P} \subseteq[\omega]^{\aleph_{0}}$ is a filter. We say $\mathcal{P}$ is a $P$-filter iff $\mathcal{P}$ contains all co-finite subsets of $\omega$, and $\left(\forall \mathcal{U} \in[\mathcal{P}]^{\aleph_{0}}\right)(\exists A \in \mathcal{P})(\forall B \in \mathcal{U})\left(A \subseteq \subseteq^{*} B\right)$.

Definition 2.4. Suppose $\mathcal{P}$ is a $P$-filter and $P$ is a forcing notion. We say that $P$ is weakly $\mathcal{P}$-bounding iff $\mathbf{1} \|_{P}$ " $\left(\forall A \in[\omega]^{\aleph_{0}}\right)(\exists B \in \mathcal{P})\left(A \nsubseteq^{*} B\right)$."

The following Theorem is [9, Conclusion VI.3.17(1)].

Theorem 2.5. Suppose $\kappa$ is a limit ordinal and $\mathcal{P}$ is a $P$-filter and $\left\langle P_{\eta}: \eta \leq \kappa\right\rangle$ is a countable support forcing iteration based on $\left\langle Q_{\eta}: \eta<\kappa\right\rangle$. Suppose for every $\eta<\kappa$ we have $P_{\eta}$ is weakly $\mathcal{P}$-bounding and $\mathbf{1} \|_{P_{\eta}}$ " $Q_{\eta}$ is proper." Then $P_{\kappa}$ is weakly $\mathcal{P}$-bounding.

Proof: This is clear if $\kappa$ has uncountable cofinality, so assume $\operatorname{cf}(\kappa)=\omega$.

Suppose $p \in P_{\kappa}$ and $A$ is a $P_{\kappa}$-name and $p \leftarrow^{\prime \prime} A \in[\omega]^{\aleph_{0}}$." Let $\lambda$ be a sufficiently large regular cardinal and $N$ a countable elementary substructure of $H_{\lambda}$ such that $\left\{P_{\kappa}, \mathcal{P}, A, p\right\} \in N$.

Let $\left\langle\alpha_{k}: k \in \omega\right\rangle \in N$ be an increasing sequence cofinal in $\kappa$ such that $\alpha_{0}=0$. Fix $B \in \mathcal{P}$ such that $(\forall X \in \mathcal{P} \cap N)\left(B \subseteq^{*} X\right)$. It suffices to show $p \nvdash \leftarrow^{\prime} A \subseteq^{*} B$."

Build $\left\langle q_{k}, p_{k}, m_{k}: k \in \omega\right\rangle$ such that $q_{0}=p$ and for every $k \in \omega$ we have that each of the following holds:

(1) $p_{k} \in P_{\alpha_{k}}$ is $N$-generic, and

(2) $p_{k} \Vdash$ " $q_{k+1} \in P_{\alpha_{k}, \kappa} \cap N\left[G_{P_{\alpha_{k}}}\right]$ and $q_{k+1} \leq q_{k} \uparrow\left[\alpha_{k}, \kappa\right)$," and

(3) $m_{k}$ is a $P_{\alpha_{k}}$-name for an integer and $p_{k} \Vdash$ "if $k>0$ then $m_{k}>m_{k-1}$," and

(4) $p_{k} \Vdash$ " $q_{k+1} \Vdash$ ' $m_{k} \notin B$ and $m_{k} \in A$,'” and

(5) $p_{k+1} \uparrow \alpha_{k}=p_{k}$, and

(6) $p_{k} \Vdash$ " $p_{k+1} \uparrow\left[\alpha_{k}, \alpha_{k+1}\right) \leq q_{k+1} \uparrow \alpha_{k+1} . "$

The construction proceeds as follows. Given $p_{k}, q_{k}$, and $m_{k-1}$, work in $V\left[G_{P_{\alpha_{k}}}\right]$ with $p_{k} \in G_{P_{\alpha_{k}}}$.

Build $A_{k} \in[\omega]^{\aleph_{0}} \cap N\left[G_{P_{\alpha_{k}}}\right]$ and $\left\langle q_{k}^{i}: i \in \omega\right\rangle \in N\left[G_{P_{\alpha_{k}}}\right]$ such that $q_{k}^{0}=$ $q_{k} \uparrow\left[\alpha_{k}, \kappa\right)$, and for every $i \in \omega$ we have $q_{k}^{i+1} \leq q_{k}^{i}$, and $q_{k}^{i+1} \Vdash " i \in A$ iff $i \in A_{k}$."

Using the hypothesis on $P_{\alpha_{k}}$ we may choose $B_{k} \in \mathcal{P}$ such that $A_{k} \mathbb{}^{*} B_{k}$. By elementarity we may assume $B_{k} \in N\left[G_{P_{\alpha_{k}}}\right]$. Because $p_{k}$ is $N$-generic, we have $B_{k} \in V \cap N\left[G_{P_{\alpha_{k}}}\right]=N$. 
Because $B_{k} \in N$ we have $B \subseteq^{*} B_{k}$, and hence $A_{k} \nsubseteq^{*} B$. Therefore we may choose $m_{k} \in \omega$ such that $m_{k} \in A_{k}$ and $m_{k} \notin B$ and if $k>0$ then $m_{k}>m_{k-1}$.

Let $q_{k+1}=q_{k}^{m_{k}+1}$. Clearly (2), (3), and (4) are satisfied.

Using the Proper Iteration Lemma we may choose $p_{k+1}$ satisfying (1), (5), and (6).

This completes the recursive construction.

Let $r \in P_{\kappa}$ be such that for every $k \in \omega$ we have $r \uparrow \alpha_{k}=p_{k}$.

Suppose, towards a contradiction, that $r^{\prime} \leq r$ and $r^{\prime} \| \leftarrow^{\text {" } A \subseteq}$. B." Fix $P_{\kappa^{-}}$ names $n$ and $k$ such that $r^{\prime} \Vdash " A \subseteq n \cup B$, and $m_{k}>n$."

By strengthening $r^{\prime}$ we may assume that $k$ and $m_{k}$ are integers rather than merely names.

Because $r^{\prime} \leq\left(p_{k+1}, q_{k+1}\right)$ we have $r^{\prime} \Vdash$ " $m_{k} \in A-n \subseteq B$, and $m_{k} \notin B$." This is a contradiction.

The Theorem is established.

Lemma 2.8. Suppose $P$ is weakly $\mathcal{P}$-bounding and $\mathbf{1} \|_{P}$ " $Q$ is almost $\mathcal{P}$ bounding." Then $P * Q$ is weakly $\mathcal{P}$-bounding.

Proof: Suppose $(p, q) \Vdash_{P * Q}$ "A $A \in[\omega]^{\aleph_{0}}$." Take $q^{\prime}$ and $B$ in $V^{P}$ such that $p \Vdash " B \in \mathcal{P}$ and $q^{\prime} \leq q$ and

$\left(^{*}\right) q^{\prime} \|{ }^{\prime}\left(\forall Y \in[\omega]^{\aleph_{0}} \cap V\left[G_{P}\right]\right)\left(A \cap Y \mathbb{E}^{*} B\right) . '$,

Take $p^{\prime} \leq p$ and $B^{\prime} \in \mathcal{P}$ such that $p^{\prime} \Vdash$ " $B \mathbb{}^{*} B^{\prime}$." By $(*)$ we have $\left(p^{\prime}, q^{\prime}\right) \Vdash$ " $A \cap\left(B-B^{\prime}\right) \nsubseteq^{*} B$." Hence $\left(p^{\prime}, q^{\prime}\right) \Vdash$ " $A \nsubseteq^{*} B^{\prime}$."

The Lemma is established.

Theorem 2.9. Suppose $\mathcal{P}$ is a $P$-filter and $\left\langle P_{\eta}: \eta \leq \kappa\right\rangle$ is a countable support forcing iteration based on $\left\langle Q_{\eta}: \eta<\kappa\right\rangle$. Suppose for every $\eta<\kappa$ we have $\mathbf{1} \|_{P_{\eta}}$ " $Q_{\eta}$ is proper and almost $\mathcal{P}$-bounding." Then $P_{\kappa}$ is weakly $\mathcal{P}$-bounding.

Proof: By Theorem 2.5 and Lemma 2.8.

\section{Preservation of weakly ${ }^{\omega} \omega$-bounding}

In this section we give an exposition of a preservation theorem, due to Shelah, concerning the weak ${ }^{\omega} \omega$-bounding property.

Definition 3.1. Suppose $f$ and $g$ are in ${ }^{\omega} \omega$. We say $f \leq^{*} g$ iff $(\exists n \in \omega)(\forall k>n)$ $(f(k) \leq g(k))$. 
Definition 3.2. Suppose $F \subseteq{ }^{\omega} \omega$ and $g \in{ }^{\omega} \omega$. We say that $g$ bounds $F$ iff $(\forall f \in F)\left(f \leq^{*} g\right)$.

Definition 3.3. Suppose $P$ is a forcing notion. We say that $P$ is weakly ${ }^{\omega} \omega$ bounding iff $\mathbf{1} \Vdash$ " $\left(\forall f \in{ }^{\omega} \omega\right)\left(\exists g \in{ }^{\omega} \omega \cap V\right)\left(g \not \mathbb{Z}^{*} f\right) . "$

The following Theorem is [9, Conclusion VI.3.17(2)].

Theorem 3.4. Suppose $\kappa$ is a limit ordinal and $\left\langle P_{\eta}: \eta \leq \kappa\right\rangle$ is a countable support forcing iteration based on $\left\langle Q_{\eta}: \eta<\kappa\right\rangle$. Suppose for every $\eta<\kappa$ we have $P_{\eta}$ is weakly ${ }^{\omega} \omega$-bounding and $\mathbf{1} \|_{P_{\eta}}$ " $Q_{\eta}$ is proper." Then $P_{\kappa}$ is weakly ${ }^{\omega} \omega$-bounding.

Proof: Use the proof of Theorem 2.5 with $\left([\omega]^{\aleph_{0}}, \mathcal{P}, \supseteq^{*}\right)$ replaced with $\left({ }^{\omega} \omega,{ }^{\omega} \omega \cap\right.$ $\left.V, \leq^{*}\right)$

The Theorem is established.

The following definition is equivalent to [9, Definition VI.3.5(1)].

Definition 3.5. Suppose $P$ is a forcing notion. We say $P$ is almost ${ }^{\omega} \omega$-bounding iff $\mathbf{1} \|$ " $\left(\forall f \in{ }^{\omega} \omega\right)\left(\exists g \in{ }^{\omega} \omega \cap V\right)\left(\forall A \in[\omega]^{\aleph_{0}} \cap V\right)\left(\exists^{\infty} n \in A\right)(f(n)<g(n))$."

Lemma 3.6. Suppose $P$ is almost ${ }^{\omega} \omega$-bounding. Then $P$ is weakly ${ }^{\omega} \omega$-bounding.

Proof: Take $A=\omega$ in Definition 3.5.

Lemma 3.7. Suppose $P$ is weakly ${ }^{\omega} \omega$-bounding and $\mathbf{1} \|_{P}$ " $Q$ is almost ${ }^{\omega} \omega$ bounding." Then $P * Q$ is weakly ${ }^{\omega} \omega$-bounding.

Proof: Like Lemma 2.7 .

The Lemma is established.

Theorem 3.9. Suppose $\left\langle P_{\eta}: \eta \leq \kappa\right\rangle$ is a countable support forcing iteration based on $\left\langle Q_{\eta}: \eta<\kappa\right\rangle$. Suppose for every $\eta<\kappa$ we have $\mathbf{1} \Vdash_{P_{\eta}}$ " $Q_{\eta}$ is proper and almost ${ }^{\omega} \omega$-bounding." Then $P_{\kappa}$ is weakly ${ }^{\omega} \omega$-bounding.

Proof: By Theorem 3.4 and Lemma 3.7.

\section{Preservation of no new random reals}

We now turn our attention to the preservation of the property "no new random reals." 
Definition 4.1. For $\tau \in{ }^{<\omega} 2$, we let $U_{\tau}=\left\{\eta \in{ }^{\omega} 2: \eta\right.$ extends $\left.\tau\right\}$.

Recall that for $A \subseteq{ }^{\omega} 2$, the outer measure of $A$ is $\mu^{*}(A)=\inf \left\{\Sigma\left\{2^{-\ln (\tau)}: \tau \in\right.\right.$ $C\}: C \subseteq{ }^{<\omega_{2}}$ and $\left.A \subseteq \bigcup\left\{U_{\tau}: \tau \in C\right\}\right\} . A$ is Lebesgue measurable iff $\left(\forall \tau \in{ }^{<\omega_{2}}\right)$ $\left(\mu^{*}\left(A \cap U_{\tau}\right)+\mu^{*}\left(U_{\tau}-A\right)=\mu^{*}\left(U_{\tau}\right)\right)$, in which case we write $\mu(A)=\mu^{*}(A)$.

Definition 4.2. Suppose $A \subseteq{ }^{\omega} 2$. We say that $A$ is closed under rational translation iff $(\forall b \in A)\left(\forall b^{*}={ }_{\text {a.e. }} b\right)\left(b^{*} \in A\right)$.

The following Lemma is known as "Kolmogorov's zero-one Law."

Lemma 4.3. Suppose $A \subseteq \omega_{2}$ is closed under rational translations and suppose that $A$ is Lebesgue measurable. Then $\mu(A)=0$ or $\mu(A)=1$.

Proof: Let $\gamma=\mu(A)$ and suppose, towards a contradiction, that $0<\gamma<1$.

Claim 1. Whenever $\tau \in<^{<\omega_{2}}$ and $\tau_{0}$ and $\tau_{1}$ are the immediate successors of $\tau$, then $\mu\left(A \cap U_{\tau_{0}}\right)=\mu\left(A \cap U_{\tau_{1}}\right)$.

Proof: We have $\mu\left(A \cap U_{\tau_{0}}\right)=2^{-\ln \left(\tau_{0}\right)} \mu\left(\left\{b \in{ }^{\omega} 2: \tau_{0}{ }^{\wedge} b \in A\right\}\right)=2^{-\operatorname{lh}\left(\tau_{1}\right)} \mu(\{b \in$ $\left.\left.\omega_{2}: \tau_{1}{ }^{\wedge} b \in A\right\}\right)=\mu\left(A \cap U_{\tau_{1}}\right)$.

Claim 2: For all $\tau \in{ }^{<\omega} 2$ we have $\mu\left(A \cap U_{\tau}\right)=2^{-\operatorname{lh}(\tau)} \gamma$.

Proof: By induction on $\tau$, using Claim 1 .

Choose $\delta>\gamma$ such that $\delta^{2}<\gamma$. Choose $C \subseteq{ }^{<\omega_{2}}$ such that $A \subseteq \bigcup\left\{U_{\tau}: \tau \in C\right\}$ and $\Sigma\left\{\mu\left(U_{\tau}\right): \tau \in C\right\}<\delta$.

For each $\tau \in C$, we may, using Claim 2, choose $C_{\tau} \subseteq{ }^{<\omega} 2$ such that $A \cap U_{\tau} \subseteq$ $\bigcup\left\{U_{\eta}: \eta \in C_{\tau}\right\}$ and $\Sigma\left\{\mu\left(U_{\eta}\right): \eta \in C_{\tau}\right\}<2^{-\operatorname{lh}(\tau)} \delta$.

Let $C^{*}=\bigcup\left\{C_{\tau}: \tau \in C\right\}$.

We have that $A \subseteq \bigcup\left\{U_{\eta}: \eta \in C^{*}\right\}$ and $\Sigma\left\{\mu\left(U_{\eta}\right): \eta \in C^{*}\right\}<\delta^{2}<\gamma$.

This contradiction establishes the Lemma.

Definition 4.4. Suppose $Y \subseteq{ }^{\omega} 2$. We define $\mathrm{RT}(Y)$, the "rational translates" of $Y$, to equal $\left\{b \in{ }^{\omega} 2:\left(\exists b^{\prime} \in Y\right)\left(b^{\prime}={ }_{\text {a.e. }} b\right)\right\}$.

Definition 4.5. Suppose $y$ and $y^{\prime}$ are perfect subsets of ${ }^{\omega} 2$ of positive Lebesgue measure. We define $y \preceq y^{\prime}$ to mean $y \subseteq \operatorname{RT}\left(y^{\prime}\right)$.

Lemma 4.6. Suppose $\left\langle y_{n}: n \in \omega\right\rangle$ is a sequence of perfect subsets of ${ }^{\omega} 2$ of positive Lebesgue measure. Then there is a perfect set $y \subseteq{ }^{\omega} 2$ of positive Lebesgue measure such that $(\forall n \in \omega)\left(y \preceq y_{n}\right)$.

Proof: By Lemma 4.3 we have that $\mu\left(\mathrm{RT}\left(y_{n}\right)\right)=1$ for every $n \in \omega$. For each $n \in \omega$ let $D_{n} \subseteq{ }^{\omega} 2$ be an open set such that $\mu\left(D_{n}\right)<2^{-n-1}$ and $D_{n} \cup$ 
$\mathrm{RT}\left(y_{n}\right)={ }^{\omega} 2$. Let $C={ }^{\omega}{ }_{2}-\bigcup\left\{D_{n}: n \in \omega\right\}$. We have that $C$ is a closed set of positive measure. Let $y$ be the perfect kernel of $C$ (see [5, page 66]). We have that $y$ is a perfect set of positive measure, and for every $n \in \omega$ we have $y \subseteq C \subseteq{ }^{\omega} 2-D_{n} \subseteq \mathrm{RT}\left(y_{n}\right)$.

The Lemma is established.

Lemma 4.7. Suppose $x$ and $y$ are subsets of $\omega_{2}$. Then $x \cap \operatorname{RT}(y)=\emptyset$ iff $\mathrm{RT}(x) \cap y=\emptyset$.

Proof: Clear.

Lemma 4.8. Let $P$ be any forcing. Then $V\left[G_{P}\right] \models "\left(\forall x \in \omega^{\omega}\right)(x$ is random over $V$ iff $(\forall y \in V)(y$ is a perfect set of positive Lebesgue measure implies $x \in \mathrm{RT}(y)))$."

Proof: Work in $V\left[G_{P}\right]$. Suppose $x \in{ }^{\omega} 2$ is not random over $V$. Let $B \in V$ be a Borel set such that $x \in B$ and $\mu(B)=0$. Let $D \in V$ be an open set such that $\mu(D)<1$ and $\operatorname{RT}(B) \subseteq D$. Let $y$ be the perfect kernel of ${ }^{\omega} 2-D$. Then $y \in V$ is a perfect set of positive measure, and because $y \cap \operatorname{RT}(B)=\emptyset$, we have $\mathrm{RT}(y) \cap B=\emptyset$, and therefore $x \notin \mathrm{RT}(y)$.

In the other direction, suppose $x \in{ }^{\omega} 2$ and $y \in V$ is a perfect set of positive measure such that $x \notin \mathrm{RT}(y)$. We show that $x$ is not random over $V$. Choose $\left\langle D_{n}: n \in \omega\right\rangle \in V$ a sequence of open sets such that for every $n \in \omega$ we have $\mu\left(D_{n}\right)<1 / n$ and ${ }^{\omega} 2-\mathrm{RT}(y) \subseteq D_{n}$. Let $B=\bigcap\left\{D_{n}: n \in \omega\right\}$. We have that $B \in V$ is a Borel set of Lebesgue measure zero and $x \in B$. Therefore $x$ is not random over $V$.

The Lemma is established.

The following is [9, Lemma VI.3.18]. Notice how the argument parallels the proof of Theorem 2.5.

Theorem 4.9. Suppose $\kappa$ is a limit ordinal and $\left\langle P_{\eta}: \eta \leq \kappa\right\rangle$ is a countable support forcing iteration based on $\left\langle Q_{\eta}: \eta<\kappa\right\rangle$. Suppose for every $\eta<\kappa$ we have $\mathbf{1} \|_{P_{\eta}}$ " $Q_{\eta}$ is proper and there are no reals that are random over $V$." Then $\mathbf{1} \|_{P_{\kappa}}$ "there are no reals that are random over $V$."

Proof: For $\operatorname{cf}(\kappa)>\omega$ this is clear, so assume instead that $\operatorname{cf}(\kappa)=\omega$.

Suppose $p \in P_{\kappa}$ and $g$ is a $P_{\kappa}$-name and $p \Vdash{ }^{\prime \prime} g \in{ }^{\omega} 2$." Let $\lambda$ be a sufficiently large regular cardinal and let $N$ be a countable elementary substructure of $H_{\lambda}$ 
containing $\left\{P_{\kappa}, p, g\right\}$. Let $\left\langle\alpha_{n}: n \in \omega\right\rangle \in N$ be an increasing sequence cofinal in $\kappa$ such that $\alpha_{0}=0$.

Using Lemma 4.6, fix $y \subseteq{ }^{\omega} 2$ a perfect set of positive Lebesgue measure such that for every perfect $y^{\prime} \in N$ with $\mu\left(y^{\prime}\right)>0$ we have $y \preceq y^{\prime}$.

Build $\left\langle q_{k}, p_{k}, m_{k}: k \in \omega\right\rangle$ such that $q_{0}=p$ and for each $k \in \omega$ we have the following:

(1) $p_{k} \in P_{\alpha_{k}}$ is $N$-generic, and

(2) $p_{k+1} \uparrow \alpha_{k}=p_{k}$, and

(3) $p_{k} \Vdash " q_{k+1} \in P_{\alpha_{k}, \kappa} \cap N\left[G_{P_{\alpha_{k}}}\right]$ and $q_{k+1} \leq q_{k} \uparrow\left[\alpha_{k}, \kappa\right)$," and

(4) $p_{k} \Vdash " p_{k+1} \uparrow\left[\alpha_{k}, \alpha_{k+1}\right) \leq q_{k+1} \uparrow \alpha_{k+1}$," and

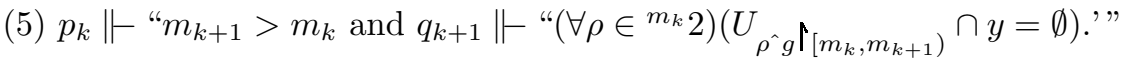

The construction proceeds as follows. Suppose we are given $p_{k}$ and $q_{k}$ and $m_{k}$. Work in $V\left[G_{P_{\alpha_{k}}}\right]$ with $p_{k} \in G_{P_{\alpha_{k}}}$. Build $\left\langle q_{k}^{i}: i \in \omega\right\rangle \in N\left[G_{P_{\alpha_{k}}}\right]$ a decreasing sequence of conditions in $P_{\alpha_{k}, \kappa}$ and $f_{k} \in{ }^{\omega} 2 \cap N\left[G_{P_{\alpha_{k}}}\right]$ such that $q_{k}^{0} \leq q_{k}$ and for every $i \in \omega$ we have $q_{k}^{i} \Vdash$ " $f_{k}(i)=g(i)$." Using the hypothesis on $P_{\alpha_{k}}$ and Lemma 4.8, we may choose a perfect set $y_{k} \in V$ of positive measure such that $f_{k} \notin \mathrm{RT}\left(y_{k}\right)$. By elementarity we may assume $y_{k} \in N\left[G_{P_{\alpha_{k}}}\right] \cap V=N$.

Because $y \preceq y_{k}$ we have $\mathrm{RT}(y) \subseteq \mathrm{RT}\left(y_{k}\right)$, and hence $f_{k} \notin \mathrm{RT}(y)$. Hence by Lemma 4.7 we have $\operatorname{RT}\left(\left\{f_{k}\right\}\right) \cap y=\emptyset$. Hence for each $\rho \in{ }^{m_{k}} 2$, we may let $m_{k}^{\rho}$

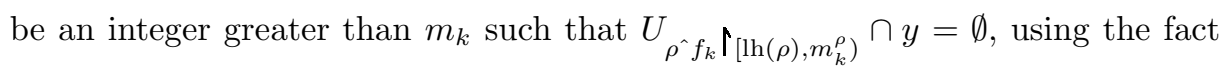
that $y$ is closed.

Let $m_{k+1}=\max \left\{m_{k}^{\rho}: \rho \in m_{k} 2\right\}$. Let $q_{k+1}=q_{k}^{m_{k+1}+1}$. We have that $q_{k+1}$ satisfies (3) and (5). Using the Proper Iteration Lemma, we may choose $p_{k+1}$ satisfying (1), (2), and (4).

This completes the recursive construction.

Let $r \in P_{\kappa}$ be chosen such that $(\forall k \in \omega)\left(r\left\lceil\alpha_{k}=p_{k}\right)\right.$.

We have $r \|$ "RT $(\{g\}) \cap y=\emptyset$." Hence by Lemmas 4.7 and 4.8 we have $r \|$ " $g$ is not random over $V$."

The Theorem is established.

\section{Preservation of "every new dense open set con- tains an old dense open set"}

In this section we prove preservation of the property "every new dense open set contains an old dense open set." Shelah includes two very different proofs of this 
fact in his book; we follow the proof given in [9, Section XVIII.3].

Throughout this section we fix an enumeration $\left\langle\eta_{n}^{*}: n \in \omega\right\rangle$ of ${ }^{<\omega} \omega$ such that whenever $\eta_{i}^{*}$ is an initial segment of $\eta_{j}^{*}$ then $i \leq j$. Also, throughout this section we let $\mathcal{B}$ equal the set of functions from $<\omega \omega$ into ${ }^{<\omega} \omega$.

Definition 5.1. Suppose $f$ and $g$ are in $\mathcal{B}$. We say $f \leq_{\mathcal{B}} g$ iff for every $\eta \in{ }^{<\omega} \omega$ there is $\nu \in{ }^{<\omega} \omega$ such that $\nu^{\wedge} f(\nu)$ is an initial segment of $\eta^{\wedge} g(\eta)$.

We remark that Definition 5.1 differs from [9, Context and Definition XVIII.3.7A] because we have incorporated [9, Remark XVIII.3.7F(1)].

Lemma 5.2. The relation $\leq_{\mathcal{B}}$ is a partial ordering of $\mathcal{B}$.

Proof: Immediate.

Lemma 5.3. Suppose $\left\langle f_{i}: i \in \omega\right\rangle$ is a sequence of elements of $\mathcal{B}$. Then there is $g \in \mathcal{B}$ such that for every $i \in \omega$ we have $f_{i} \leq_{\mathcal{B}} g$.

Proof: For every $\eta \in{ }^{<\omega} \omega$ and $k \in \omega$ define $g_{0}(\eta)=\eta$ and $g_{k+1}(\eta)=$ $g_{k}(\eta)^{\wedge} f_{k}\left(\eta^{\wedge} g_{k}(\eta)\right)$. Define $g(\eta)$ to equal $g_{n}(\eta)$ where $\eta=\eta_{n}^{*}$.

To see that $f_{k} \leq_{\mathcal{B}} g$ it suffices to note that whenever $n>k$ then

$$
\left(\eta_{n}^{* \uparrow} g_{k}\left(\eta_{n}^{*}\right)\right)^{\uparrow} f_{k}\left(\eta_{n}^{*} \widehat{g}_{k}\left(\eta_{n}^{*}\right)\right)=\eta_{n}^{* \uparrow} g_{k+1}\left(\eta_{n}^{*}\right) \subseteq \eta_{n}^{* \uparrow} g\left(\eta_{n}^{*}\right)
$$

The Lemma is established.

Lemma 5.4. Suppose $P$ is a forcing notion. Then every dense open subset of $\omega_{\omega} \omega$ in $V\left[G_{P}\right]$ contains a dense open subset of $\omega_{\omega}$ in $V$ iff $V\left[G_{P}\right] \models "(\forall f \in \mathcal{B})$ $(\exists g \in \mathcal{B} \cap V)\left(f \leq_{\mathcal{B}} g\right) . "$

Proof: We first establish the "if" direction. Work in $V\left[G_{P}\right]$. Suppose $D$ is a dense open subset of ${ }^{\omega} \omega$. Pick $f \in \mathcal{B}$ such that for every $\eta \in{ }^{<\omega} \omega$ we have $U_{\eta^{\wedge} f(\eta)} \subseteq D$. Fix $g \in \mathcal{B} \cap V$ such that $f \leq_{\mathcal{B}} g$. Let $D^{\prime}=\bigcup\left\{U_{\eta^{\wedge} g(\eta)}: \eta \in{ }^{<\omega} \omega\right\}$. We have $D^{\prime}$ is a dense open subset of $D$ and $D^{\prime} \in V$.

For the "only if" direction, suppose $f \in \mathcal{B}$. Build $\left\langle D_{n}, \eta_{n}, x_{n}: n \in \omega\right\rangle$ recursively such that for every $n \in \omega$ we have that either $U_{\eta_{n}^{*}} \subseteq \bigcup\left\{D_{i}: i<n\right\}$ and $D_{n}=D_{n-1}$ and $x_{n}=x_{n-1}$ and $\eta_{n}=\eta_{n-1}$, or all of the following::

(1) $\eta_{n}$ extends $\eta_{n}^{*}$, and

(2) $D_{n}=U_{\eta_{n} \wedge f\left(\eta_{n}\right)^{\wedge}\langle 0\rangle}$, and

(3) $D_{n}$ is disjoint from $\bigcup\left\{D_{i}: i<n\right\} \cup\left\{x_{i}: i<n\right\}$, and

(4) $x_{n} \in{ }^{\omega} \omega$ extends $\eta_{n} \wedge f\left(\eta_{n}\right)^{\wedge}\langle 1\rangle$. 
We may take $D^{\prime} \in V$ open dense such that $D^{\prime} \subseteq \bigcup\left\{D_{n}: n \in \omega\right\}$.

Choose $g \in \mathcal{B} \cap V$ such that $\left(\forall \eta \in{ }^{<\omega} \omega\right)\left(U_{\eta^{\wedge} g(\eta)} \subseteq D^{\prime}\right)$.

Given $\eta \in{ }^{<\omega} \omega$, pick $n \in \omega$ such that $U_{\eta^{\wedge} g(\eta)} \cap U_{\eta_{n} \wedge f\left(\eta_{n}\right)^{\wedge}\langle 0\rangle} \neq \emptyset$. We have $x_{n} \notin D^{\prime}$, and so $U_{\eta^{\wedge} g(\eta)} \subseteq U_{\eta_{n} \wedge f\left(\eta_{n}\right)}$. It follows that $f \leq_{\mathcal{B}} g$.

The Lemma is established.

The following is [9, Conclusion VI.2.15D] and [9, Claim XVIII.3.7D]; we follow the proof given in $[9$, Chapter XVIII]

Theorem 5.5. Suppose $\left\langle P_{\eta}: \eta \leq \kappa\right\rangle$ is a countable support forcing iteration based on $\left\langle Q_{\eta}: \eta<\kappa\right\rangle$. Suppose for every $\eta<\kappa$ we have $\mathbf{1} \vdash_{P_{\eta}}$ " $Q_{\eta}$ is proper and $\mathbf{1} \|_{Q_{\eta}}$ 'for every dense open $D \subseteq{ }^{\omega} \omega$ there is a dense open $D^{\prime} \subseteq D$ such that $D^{\prime} \in V\left[G_{P_{\eta}}\right]$." "Then $\mathbf{1} \|_{P_{\kappa}}$ "for every dense open $D \subseteq{ }^{\omega} \omega$ there is a dense open $D^{\prime} \subseteq D$ such that $D^{\prime} \in V$."

Proof: By induction on $\kappa$. The induction step is clear for $\kappa$ a successor ordinal and, in light of Lemma 5.4, it is likewise clear for $\kappa$ of uncountable cofinality. So we assume $\operatorname{cf}(\kappa)=\omega$.

Suppose $p \in P_{\kappa}$ and $f$ is a $P_{\kappa}$-name and $p \Vdash$ " $f \in \mathcal{B}$." Choose $\lambda$ a sufficiently large regular cardinal and $N$ a countable elementary substructure of $H_{\lambda}$ such that $\left\{P_{\kappa}, f, p\right\} \in N$.

Let $\left\langle\alpha_{k}: k \in \omega\right\rangle \in N$ be an increasing sequence cofinal in $\kappa$ such that $\alpha_{0}=0$. Using Lemma 5.3, fix $g \in \mathcal{B}$ such that $(\forall h \in \mathcal{B} \cap N)\left(h \leq_{\mathcal{B}} g\right)$.

Build $\left\langle q_{k}, p_{k}, m_{k}: k \in \omega\right\rangle$ such that $q_{0}=p$ and for every $k \in \omega$ we have that each of the following holds:

(1) $p_{k} \in P_{\alpha_{k}}$ is $N$-generic, and

(2) $p_{k} \|$ " $q_{k+1} \in P_{\alpha_{k}, \kappa} \cap N\left[G_{P_{\alpha_{k}}}\right]$ and $q_{k+1} \leq q_{k} \uparrow\left[\alpha_{k}, \kappa\right)$," and

(3) $p_{k} \Vdash$ " $q_{k+1} \Vdash{ }^{\prime} m_{k} \in \omega$ and $\eta_{k}^{* \wedge} g\left(\eta_{k}^{*}\right)$ extends $\eta_{m_{k}}^{*}{ }^{\wedge} f\left(\eta_{m_{k}}^{*}\right)$,'” and

(4) $p_{k+1} \uparrow \alpha_{k}=p_{k}$, and

(5) $p_{k} \Vdash$ " $p_{k+1} \uparrow\left[\alpha_{k}, \alpha_{k+1}\right) \leq q_{k+1} \uparrow \alpha_{k+1}$."

The construction proceeds as follows. Given $p_{k}$ and $q_{k}$, work in $V\left[G_{P_{\alpha_{k}}}\right]$ with $p_{k} \in G_{P_{\alpha_{k}}}$.

Build $\left\langle q_{k}^{i}: i \in \omega\right\rangle \in N\left[G_{P_{\alpha_{k}}}\right]$ and $f_{k} \in \mathcal{B} \cap N\left[G_{P_{\alpha_{k}}}\right]$ such that $q_{k}^{0}=q_{k} \uparrow\left[\alpha_{k}, \kappa\right)$, and for every $i \in \omega$ we have the following:

(1) $q_{k}^{i+1} \leq q_{k}^{i}$, and

(2) $q_{k}^{i+1} \Vdash$ " $f_{k}\left(\eta_{i}^{*}\right)=f\left(\eta_{i}^{*}\right)$." 
Using Lemma 5.4, choose $g_{k} \in \mathcal{B} \cap V$ such that $f_{k} \leq_{\mathcal{B}} g_{k}$. We may assume $g_{k} \in N\left[G_{P_{\alpha_{k}}}\right]$. Hence $g_{k} \in N$. Hence $g_{k} \leq_{\mathcal{B}} g$.

By Lemma 5.2 we have $f_{k} \leq_{\mathcal{B}} g$, so we may choose $m_{k}$ such that $\eta_{k}^{* \wedge} g\left(\eta_{k}^{*}\right)$ extends $\eta_{m_{k}}^{*}{ }^{\wedge} f_{k}\left(\eta_{m_{k}}^{*}\right)$.

Let $q_{k+1}=q_{k}^{m_{k}+1}$. We have that $q_{k+1}$ and $m_{k}$ satisfy (2) and (3).

Using the Proper Iteration Lemma we may choose $p_{k+1}$ satisfying (1), (4), and (5).

This completes the recursive construction.

Let $r \in P_{\kappa}$ be such that for every $k \in \omega$ we have $r\left\lceil\alpha_{k}=p_{k}\right.$.

Suppose, towards a contradiction, that $r^{\prime} \leq r$ and $r^{\prime} \Vdash " f \mathbb{Z}_{\mathcal{B}} g$." Fix a $P_{\kappa}$-name $k$ such that $r^{\prime} \Vdash$ " $\eta_{k}^{* \wedge} g\left(\eta_{k}^{*}\right)$ does not extend $\eta_{m_{k}}^{*}{ }^{\wedge} f\left(\eta_{m_{k}}^{*}\right)$."

By strengthening $r^{\prime}$ we may assume that $k$ and $m_{k}$ are integers rather than names.

Because $r^{\prime} \leq\left(p_{k+1}, q_{k+1}\right)$ we have $r^{\prime} \Vdash{ }^{\prime \prime} \eta_{k}^{* \wedge} g\left(\eta_{k}^{*}\right)$ extends $\eta_{m_{k}}^{*}{ }^{\wedge} f\left(\eta_{m_{k}}^{*}\right)$." This is a contradiction.

The Theorem is established.

\section{On "the set of reals that are in the ground model has positive outer measure in the forc- ing extension"}

In this section we present a theorem of Shelah ([9, Claim XVIII.3.8B(3)]) that gives a sufficient condition for a forcing iteration to satisfy $\mu^{*}\left(\omega_{2} \cap V\right)>0$. This notion has been investigated also by [4].

Definition 6.1. We let $\mathcal{B}^{\prime}$ be the set of functions $f$ from $\omega$ into ${ }^{<\omega} 2$ such that $\Sigma\left\{\mu\left(U_{f(m)}\right): m \in \omega\right\} \leq 1$.

Lemma 6.2. Suppose $g \in{ }^{\omega} 2$ and $\lambda$ is a sufficiently large regular cardinal and $N$ is a countable elementary substructure of $H_{\lambda}$. Then $g$ is random over $N$ iff $\left(\forall f \in \mathcal{B}^{\prime} \cap N\right)(\exists m \in \omega)(\forall i \geq m)(g$ does not extend $f(i))$.

Proof: We first establish the "only if" direction. Suppose $g \in{ }^{\omega} 2$ and $f \in \mathcal{B}^{\prime} \cap N$ and $\left(\exists^{\infty} m \in \omega\right)(g$ extends $f(m))$. Let $B=\left\{h \in{ }^{\omega} 2:\left(\exists^{\infty} m \in \omega\right)(f(m)\right.$ is an initial segment of $h)\}$. Then $B \subseteq{ }^{\omega} 2$ is a Borel set and $g \in B \in N$, and $\mu(B)=0$ because for every $n \in \omega$ we have that $B$ is covered by $\bigcup\left\{U_{f(i)}: i \geq n\right\}$, and $\lim _{n \rightarrow \infty}\left(\mu\left(\bigcup\left\{U_{f(i)}: i \geq n\right\}\right)=0\right.$. Therefore $g$ is not random over $N$. 
To prove the "if" direction, suppose that $g$ is not random over $N$. We may choose $B \in N$ a Borel set of measure zero such that $g \in B \in N$. Let $\left\langle D_{n}: n \in\right.$ $\omega\rangle \in N$ be a sequence of open subsets of ${ }^{\omega} 2$ such that for every $n \in \omega$ we have $B \subseteq D_{n}$ and $\mu\left(D_{n}\right)<2^{-n}$. For each $n \in \omega$ choose $k_{n} \leq \omega$ and $\left\langle\eta_{i}^{n}: i<k_{n}\right\rangle$ a sequence of pairwise incomparable elements of ${ }^{<\omega} 2$ such that $D_{n}=\bigcup\left\{U_{\eta_{i}^{n}}\right.$ : $\left.i<k_{n}\right\}$. Furthermore we may assume that $\left\langle\left\langle\eta_{i}^{n}: i<k_{n}\right\rangle: n \in \omega\right\rangle$ is an element of $N$. Let $f \in N$ be a one-to-one function mapping $\omega$ onto $\left\{\eta_{i}^{n}: i<k_{n}\right.$ and $n \in \omega\}$. Then we have that $f \in \mathcal{B}^{\prime}$ and $\left(\exists^{\infty} m \in \omega\right)\left(g \in U_{f(m)}\right)$. The Lemma is established.

Lemma 6.3. Suppose $g \in{ }^{\omega} 2$ and $\lambda$ is a sufficiently large regular cardinal and $N$ is a countable elementary substructure of $H_{\lambda}$. Suppose $g$ is random over $N$. Suppose $Y \in N$ is a subset of ${ }^{<\omega} 2$ and $\Sigma\left\{\mu\left(U_{\eta}\right): \eta \in Y\right\}$ is finite. Then $\{\eta \in Y$ : $\left.g \in U_{\eta}\right\}$ is finite.

Proof: We may assume $Y$ is infinite. Choose a finite integer $m$ and infinite sets (not necessarily disjoint) $D_{i} \subseteq Y$ for $i<m$ such that each $D_{i}$ is in $N$ and $\bigcup\left\{D_{i}\right.$ : $i<m\}=Y$ and for each $i<m$ we have $\Sigma\left\{\mu\left(U_{\eta}\right): \eta \in D_{i}\right\} \leq 1$. For each $i<m$ choose $f_{i} \in N$ such that $f_{i}$ maps $\omega$ onto $D_{i}$. By Lemma 6.2, for every $i<m$ there is $\beta_{i} \in \omega$ such that $\left(\forall j \geq \beta_{i}\right)\left(g\right.$ does not extend $\left.f_{i}(j)\right)$. Hence $\{\eta \in Y$ : $\left.g \in U_{\eta}\right\} \subseteq \bigcup\left\{\left\{f_{i}(j): j<\beta_{i}\right\}: i<m\right\}$, which is finite. The Lemma is established.

Lemma 6.4. Suppose $P$ is a poset such that whenever $\lambda$ is a sufficiently large regular cardinal and $N$ is a countable elementary substructure of $H_{\lambda}$ and $P \in N$ and $g \in{ }^{\omega} 2$ and $g$ is random over $N$, then $V\left[G_{P}\right] \models$ " $g$ is random over $N\left[G_{P}\right]$." Then $V\left[G_{P}\right] \models " \omega 2 \cap V$ has positive outer measure."

Proof: Suppose, towards a contradiction, that in $V\left[G_{P}\right]$ we have that $B$ is a Borel subset of ${ }^{\omega} 2$ such that ${ }^{\omega} 2 \cap V \subseteq B$ and $\mu(B)=0$.

In $V$, choose $\lambda$ a sufficiently large regular cardinal and $N$ a countable elementary substructure of $H_{\lambda}$ such that $p \in N$ and a name for $B$ is in $N$. Let $g \in{ }^{\omega} 2$ be random over $N$. By hypothesis, $V\left[G_{P}\right] \models$ "g is random over $N\left[G_{P}\right] . "$ Therefore $V\left[G_{P}\right] \models " g \notin B$." This contradiction establishes the Lemma.

Lemma 6.5. Suppose $P$ is a poset. Suppose $\chi$ is a sufficiently large regular cardinal and $\lambda$ is a regular cardinal sufficiently larger than $\chi$. Suppose $N$ is a countable elementary substructure of $H_{\lambda}$ and $N_{1}$ and $N_{2}$ are countable elementary substructures of $H_{\chi}$ and $\chi \in N$ and $P \in N_{1} \in N_{2} \in N$. Suppose also 
(1) $G_{1} \subseteq P \cap N_{1}$ is an $N_{1}$-generic subset of $P$, and

(2) $p \in G_{1}$ and $G_{1} \in N$, and

(3) $\left\langle f_{l}: l \leq k\right\rangle \in N$ is a finite sequence of $P$-names such that $p \|$ " $f_{l} \in \mathcal{B}^{\prime} \cap$ $N_{1}\left[G_{P}\right] "$ for all $l \leq k$, and

(4) $g \in \omega^{\omega} 2$ is random over $N$, and

(5) $\left\langle\beta_{l}: l \leq k\right\rangle$ is a sequence of integers and for all $l \leq k$ we have $\left(\forall j \geq \beta_{l}\right)(g$ does not extend $\left.f_{l}\left[G_{1}\right](j)\right)$. That is, for every $j \geq \beta_{l}$ there is $p^{\prime} \in G_{1}$ and $\rho \in{ }^{<\omega} 2$ such that $g$ does not extend $\rho$ and $p^{\prime} \|$ " $\rho=f_{l}(j)$."

Then there is $G_{2} \subseteq P \cap N_{2}$ an $N_{2}$-generic subset of $P$ such that $p \in G_{2}$ and $G_{2} \in N$ and for all $l \leq k$ we have $\left(\forall j \geq \beta_{l}\right)\left(g\right.$ does not extend $\left.f_{l}\left[G_{2}\right](j)\right)$.

Proof: Build $\left\langle p_{n}: n \in \omega\right\rangle \in N$ and $\left\langle m_{n}: n \in \omega\right\rangle \in N$ and $\left\langle f_{l}^{*}: l \leq k\right\rangle \in N$ such that $p_{0}=p$ and for each $n \in \omega$ we have each of the following:

(1) $p_{n} \in G_{1}$ and $p_{n+1} \leq p_{n}$, and

(2) $m_{n}$ is an integer such that $m_{n} \geq n$ and $p_{n} \|$ " $\Sigma\left\{\mu\left(U_{f_{l}(i)}\right): i \geq m_{n}\right\}<2^{-n}$ for each $l \leq k, "$ and

(3) for every $l \leq k$ we have $f_{l}^{*} \in N$ maps $\omega$ into ${ }^{<\omega} 2$, and

(4) $p_{n} \Vdash$ " $f_{l} \uparrow m_{n}=f_{l}^{*} \uparrow m_{n}$ for each $l \leq k$."

Claim 1. For $l \leq k$ we have $f_{l}^{*} \in \mathcal{B}^{\prime}$.

Proof. Suppose, towards a contradiction, that $l \leq k$ and $m \in \omega$ and $\Sigma\left\{\mu\left(U_{f_{l}^{*}(i)}\right)\right.$ : $i<m\}>1$. Because $p_{m} \Vdash$ " $f_{l} \uparrow m=f_{l}^{*} \uparrow m$," it follows that $p_{m} \Vdash$ " $f_{l} \notin \mathcal{B}^{\prime}$." This contradiction establishes the Claim.

Build $\left\langle p_{n, m}: m \in \omega, n \in \omega\right\rangle \in N$ and $\left\langle f_{l, n}^{*}: l \leq k, n \in \omega\right\rangle \in N$ such that each of the following holds:

(1) for every $n \in \omega$ we have that $\left\langle p_{n, m}: m \in \omega\right\rangle$ is an $N_{2}$-generic sequence for $P$ and $p_{n, 0}=p_{n}$, and

(2) for every $l \leq k$ and $n \in \omega$ and $m \in \omega$ we have $p_{n, m} \Vdash$ " $f_{l, n}^{*} \uparrow m=f_{l} \uparrow m$."

Claim 2. For $l \leq k$ and $n \in \omega$ we have $f_{l, n}^{*} \in \mathcal{B}^{\prime}$.

Proof: Similar to Claim 1.

Claim 3. For every $l \leq k$ and $n \in \omega$ we have $\Sigma\left\{\mu\left(U_{f_{l, n}^{*}(i)}\right): i \geq m_{n}\right\} \leq 2^{-n}$.

Proof: Suppose $l$ and $n$ constitute a counterexample. Then we can choose an integer $t$ so large that $\Sigma\left\{\mu\left(U_{f_{l, n}^{*}(i)}\right): m_{n} \leq i<t\right\}>2^{-n}$. We have $p_{n, t} \Vdash$ " $\Sigma\left\{\mu\left(U_{f_{l, n}^{*}(i)}\right): m_{n} \leq i<t\right\}=\Sigma\left\{\mu\left(U_{f_{l}^{*}(i)}\right): m_{n} \leq i<t\right\}<\Sigma\left\{\mu\left(U_{f_{l}^{*}(i)}\right): m_{n} \leq\right.$ $i<\omega\}<2^{-n}$." This contradiction establishes the Claim. 
For each $l \leq k$ and $n \in \omega$ let $U_{l, n}^{*}=\bigcup\left\{U_{f_{l, n}^{*}(i)}: i \in \omega\right\}$.

Claim 4. For every $l \leq k$ and $n \in \omega$ we have $U_{l, n}^{*} \subseteq \bigcup\left\{U_{f_{l}^{*}(i)}: i \in \omega\right\} \cup$ $\bigcup\left\{U_{f_{l, n}^{*}(i)}: i \geq m_{n}\right\}$.

Proof: The Claim is forced by the condition $p_{n, n}$, hence it is true outright.

For each $l \leq k$ let $U_{l}^{*}=\bigcup\left\{U_{l, n}^{*}: n \in \omega\right\}$. By Claims 3 and 4 we have that $\mu\left(U_{l}^{*}\right)$ is finite for every $l \leq k$. By Lemma 6.4 we have that $\left\{\rho \in{ }^{<\omega} 2:(\exists l \leq k)(\exists n \in \omega)\right.$ $(\exists i \in \omega)\left(\rho=f_{l, n}^{*}(i)\right.$ and $g$ extends $\left.\left.\rho\right)\right\}$ is finite. Therefore, we may fix $n^{*}$ so large that $(\forall l \leq k)(\forall n \in \omega)(\forall i \in \omega)\left(g\right.$ extends $f_{l, n}^{*}(i)$ only if $\left.\mu\left(U_{f_{l, n}^{*}(i)}\right) \geq 2^{-n^{*}}\right)$.

Claim 5. Suppose $l \leq k$ and $i \in \omega$ and $n \in \omega$ and $\mu\left(U_{f_{l, n}^{*}(i)}\right) \geq 2^{-n^{*}}$. Then $i<m_{n^{*}}$.

Proof: Suppose $i \geq m_{n^{*}}$. Then $p_{n, i+1} \|$ “ $\mu\left(U_{f_{l, n}^{*}(i)}\right)=\mu\left(U_{f_{l}(i)}\right)<\Sigma\left\{\mu\left(U_{f_{l}(j)}\right)\right.$ : $\left.j \geq m_{n^{*}}\right\}<2^{-n^{*}}$. This contradiction establishes the Claim.

Fix $t>m_{n^{*}}$ such that $t>\beta_{l}$ for every $l \leq k$. For every $l \leq k$ we have $p_{n^{*}, t} \Vdash$ " $f_{l, n^{*}}^{*} \uparrow t=f_{l}^{*} \uparrow t$." Thus, by Claim 5, we have that $p_{n^{*}, t} \Vdash$ " $(\forall l \leq k)$ $\left(\forall i \geq \beta_{l}\right)\left(g\right.$ does not extend $\left.f_{l, n^{*}}^{*}(i)\right)$."

Let $G_{2}=\left\{p^{\prime} \in P \cap N_{2}:(\exists m \in \omega)\left(p_{n^{*}, m} \leq p^{\prime}\right)\right\}$. We have that $G_{2}$ is as required.

The Lemma is established.

Definition 6.6. Suppose $g \in{ }^{\omega} 2$. We say that $P$ is $g$-good iff whenever

(1) $\chi$ is a sufficiently large regular cardinal and $\lambda$ is a regular cardinal sufficiently larger than $\chi$ and

(2) $N$ is a countable elementary substructure of $H_{\lambda}$ and $\chi \in N$ and $N_{1}$ is a countable elementary substructure of $H_{\chi}$ and

(3) $P \in N_{1} \in N$ and

(4) $g$ is random over $N$ and

(5) $k \in \omega$ and $\left\langle f_{l}: l<k\right\rangle \in N$ is a sequence of $P$-names and

(6) $p \in P \cap N_{1}$ and

(7) $p \Vdash "(\forall l<k)\left(f_{l} \in \mathcal{B}^{\prime} \cap N_{1}\left[G_{P}\right]\right)$," and

(8) $\left\langle f_{l}^{*}: l<k\right\rangle$ is a sequence of elements of $\mathcal{B}^{\prime}$ and $\left\langle\beta_{l}: l<k\right\rangle$ is a sequence of integers and for every $l<k$ we have $\left(\forall m \geq \beta_{l}\right)\left(g\right.$ does not extend $\left.f_{l}^{*}(m)\right)$ and

(9) $G_{1} \subseteq P \cap N_{1}$ and $G_{1} \in N$ and $G_{1}$ is $N_{1}$-generic over $P$ and $p \in G_{1}$ and 
(10) $(\forall l<k)\left(f_{l}\left[G_{1}\right]=f_{l}^{*}\right)$,

then there is $q \leq p$ such that $q$ is $N$-generic and $q \Vdash$ " $g$ is random over $N\left[G_{P}\right]$ and $(\forall l<k)\left(\forall m \geq \beta_{l}\right)\left(g\right.$ does not extend $\left.f_{l}(m)\right)$."

Lemma 6.7. Suppose we have that

(1) $g \in{ }^{\omega} 2$ and

(2) $\mathbf{1} \Vdash$ "Q is $g$-good," and

(3) $\chi$ is a sufficiently large regular cardinal and $\lambda$ is a regular cardinal sufficiently larger than $\chi$, and

(4) $N$ is a countable elementary substructure of $H_{\lambda}$ and $\{P * Q, \chi\} \in N$, and

(5) $p \in P$ is $N$-generic and $q$ is a $P$-name and

(6) $p \Vdash$ " $N_{1}$ is a countable elementary substructure of $H_{\chi}\left[G_{P}\right]$ and $N_{1} \in N\left[G_{P}\right]$ and $g$ is random over $N\left[G_{P}\right]$ and $q \in Q \cap N_{1}$," and

(7) $k \in \omega$ and $p \|^{\text {" }}\left\langle f_{l}: l<k\right\rangle \in N\left[G_{P}\right]$ is a sequence of $Q$-names and $q \|_{Q}$ ' $(\forall l<$ $k)\left(f_{l} \in \mathcal{B}^{\prime} \cap N_{1}\left[G_{Q}\right]\right)$.,'” and

(8) $\left\langle f_{l}^{*}: l<k\right\rangle$ and $\left\langle\beta_{l}: l<k\right\rangle$ are sequences of $P$-names and $p \Vdash "(\forall l<k)$ $\left(f_{l}^{*} \in \mathcal{B}^{\prime} \cap N\left[G_{P}\right]\right.$ and $\beta_{l} \in \omega$ and $\left(\forall i \geq \beta_{l}\right)\left(g\right.$ does not extend $\left.\left.f_{l}^{*}(i)\right)\right)$," and

(9) $G$ is a $P$-name and $p \Vdash$ " $G \subseteq Q \cap N_{1}$ is generic over $N_{1}$ and $q \in G \in N\left[G_{P}\right]$ and $(\forall l<k)\left(f_{l}^{*}=f_{l}[G]\right)$."

Then there is a $P$-name $r$ such that $p \|$ " $r \leq q$ " and $(p, r)$ is $N$-generic and $(p, r) \Vdash " g$ is random over $N\left[G_{P * Q}\right]$ and $(\forall l<k)\left(\forall i \geq \beta_{l}\right)(g$ does not extend $\left.f_{l}(i)\right)$."

Proof: Immediate.

Theorem 6.8. Suppose $g \in{ }^{\omega} 2$ and suppose $\left\langle P_{\eta}: \eta \leq \kappa\right\rangle$ is a countable support forcing iteration based on $\left\langle Q_{\eta}: \eta<\kappa\right\rangle$. Suppose for every $\eta<\kappa$ we have $\mathbf{1} \Vdash_{P_{\eta}}$ " $Q_{\eta}$ is proper and $g$-good.'" Suppose also

(1) $\chi$ is a sufficiently large regular cardinal and $\lambda$ is a regular cardinal sufficiently larger than $\chi$, and

(2) $N$ is a countable elementary substructure of $H_{\lambda}$ and $\left\{P_{\kappa}, \chi\right\} \in N$ and

(3) $\alpha \in \kappa \cap N$ and $p \in P_{\alpha} \cap N$ and 
(4) $p \|$ " $N^{\prime}$ is a countable elementary substructure of $H_{\chi}\left[G_{P_{\alpha}}\right]$ and $P_{\alpha, \kappa} \in N^{\prime} \in$ $N\left[G_{P_{\alpha}}\right]$ (so necessarily $\alpha \in N^{\prime}$ )," and

(5) $p$ is $N$-generic and $g$ is a $P_{\alpha}$-name and $p \Vdash$ " $g$ is random over $N\left[G_{P_{\alpha}}\right]$ and $q \in P_{\alpha, \kappa} \cap N^{\prime}, "$ and

(6) $k \in \omega$ and $p \| "\left\langle f_{l}: l<k\right\rangle \in N\left[G_{P_{\alpha}}\right]$ is a sequence of $P_{\alpha, \kappa}$-names and $q \|_{P_{\alpha, \kappa}}(\forall l<k)\left(f_{l} \in \mathcal{B}^{\prime} \cap N^{\prime}\left[G_{P_{\alpha, \kappa}}\right]\right), '$ ' and

(7) $\left\langle f_{l}^{*}: l<k\right\rangle$ and $\left\langle\beta_{l}: l<k\right\rangle$ are sequences of $P_{\alpha}$-names and $p \Vdash$ "( $\left.\forall l<k\right)$ $\left(f_{l}^{*} \in \mathcal{B}^{\prime} \cap N\left[G_{P_{\alpha}}\right]\right.$ and $\beta_{l} \in \omega$ and $\left(\forall i \geq \beta_{l}\right)\left(g\right.$ does not extend $\left.\left.f_{l}^{*}(i)\right)\right)$," and

(8) $G$ is a $P_{\alpha}$-name and $p \|$ " $G \subseteq P_{\alpha, \kappa} \cap N^{\prime}$ is generic over $N^{\prime}$ and $q \in G \in N\left[G_{P_{\alpha}}\right]$ and $(\forall l<k)\left(f_{l}^{*}=f_{l}[G]\right)$."

Then there is $r \in P_{\kappa}$ such that $r \uparrow \alpha=p$ and $p \|$ " $r \uparrow[\alpha, \kappa) \leq q$ " and $r$ is $N$ generic and $r \|$ " $g$ is random over $N\left[G_{P_{\kappa}}\right]$ and $(\forall l<k)\left(\forall i \geq \beta_{l}\right)(g$ does not extend $\left.f_{l}(i)\right)$."

Proof: By induction on $\kappa$.

Successor case: $\kappa=\gamma+1$.

In $V\left[G_{P_{\alpha}}\right]$ let $G_{1}=G \uparrow \gamma$ and $G_{2}=G / G_{1}$. That is, $G_{1}=\left\{p^{\prime} \uparrow \gamma: p^{\prime} \in G\right\}$ and $\left(\forall p^{\prime} \in P_{\alpha, \gamma}\right)\left(\forall r^{\prime}\right)\left(p^{\prime} \Vdash " r^{\prime} \in G_{2}\right.$ " iff $\left(\forall p^{*} \leq p^{\prime}\right)\left(\exists q^{\prime} \in G\right)\left(\exists p^{\#} \leq p^{*}\right)\left(p^{\#} \leq q^{\prime} \uparrow \gamma\right.$ and $p^{\#} \|$ " $\left.r^{\prime}=q^{\prime}(\gamma) "\right)$ ).

Choose $N^{*}$ a countable elementary substructure of $H_{\chi}\left[G_{P_{\alpha}}\right]$ such that $N^{\prime}\left[G_{P_{\alpha}}\right] \in$ $N^{*} \in N\left[G_{P_{\alpha}}\right]$ and $G \in N^{*}$. Choose $\left\langle f_{l}^{* *}: l<k\right\rangle$ such that for all $l<k$ we have $\left(p, q\lceil\gamma) \Vdash_{P_{\gamma}}\right.$ " $f_{l}^{* *}=f_{l}\left[G_{2}\right]$." Because $p \Vdash{ }^{*} f_{l}^{* *}\left[G_{1}\right]=f_{l}^{*}$ " for all $l<k$, we have that $(p, q \uparrow \gamma) \Vdash$ "( $\forall l<k)\left(\forall j \geq \beta_{l}\right)\left(g\right.$ does not extend $\left.f_{l}^{* *}(j)\right)$."

Use Lemma 6.5 to choose $G_{1}^{\prime}$ such that $p \Vdash$ " $G_{1}^{\prime} \subseteq P_{\alpha, \gamma} \cap N^{*}$ is generic over $N^{*}$ and $q \uparrow \gamma \in G_{1}^{\prime}$ and $G_{1}^{\prime} \in N\left[G_{P_{\alpha}}\right]$ and $(\forall l<k)\left(\forall j \geq \beta_{l}\right)(g$ does not extend $\left.f_{l}^{* *}\left[G_{1}^{\prime}\right](j)\right)$."

By the induction hypothesis, with $G_{1}^{\prime}$ playing the role of $G$ and $\left\langle f_{l}^{* *}: l<k\right\rangle$ playing the role of $\left\langle f_{l}: l<k\right\rangle$, we can choose $r^{\prime} \in P_{\gamma}$ such that $r^{\prime} \uparrow \alpha=p$ and $p \Vdash$ " $r^{\prime} \uparrow[\alpha, \gamma) \leq q \uparrow \gamma^{\prime}$ " and $r^{\prime}$ is $N$-generic and $r^{\prime} \Vdash$ " $g$ is random over $N\left[G_{P_{\gamma}}\right]$ and $(\forall l<k)\left(\forall i \geq \beta_{l}\right)\left(g\right.$ does not extend $\left.f_{l}^{* *}(i)\right)$."

Using Lemma 6.7 with $G_{2}$ playing the role of $G$ and $N^{\prime}\left[G_{P_{\gamma}}\right]$ playing the role of $N_{1}$, we may choose $r^{*}$ such that $r^{\prime} \|$ " $r^{*} \in Q_{\gamma}$ and $r^{*} \leq q(\gamma)$ " and $\left(r^{\prime}, r^{*}\right) \Vdash " g$ is random over $N\left[G_{P_{\kappa}}\right]$ and $(\forall l<k)\left(\forall i \geq \beta_{l}\right)\left(g\right.$ does not extend $\left.f_{l}(i)\right)$."

Let $r=\left(r^{\prime}, r^{*}\right)$. This concludes the verification of the successor case. 
Limit case: $\kappa$ is a limit ordinal.

Let $\left\langle\alpha_{n}: n \in \omega\right\rangle$ be an increasing sequence from $\kappa \cap N$ cofinal in $\sup (\kappa \cap N)$ such that $\alpha_{0}=\alpha$. Let $\left\langle\sigma_{n}: n \in \omega\right\rangle$ list all $P_{\kappa}$-names $\sigma$ such that $\sigma \in N$ and $\mathbf{1} \|_{P_{\kappa}}$ " $\sigma$ is an ordinal." Let $\left\langle f_{l}: l \in \omega\right\rangle$ be a sequence that extends $\left\langle f_{l}: l<k\right\rangle$, such that it lists the set of all $P_{\kappa}$-names $f$ in $N$ such that $(p, q) \Vdash$ " $f \in \mathcal{B}^{\prime}$."

Build $\left\langle p_{n}, q_{n}, \beta_{n}, G_{n}, G_{n}^{*}, G_{n}^{\prime}, N_{n}\right\rangle$ such that $p_{0}=p$ and $q_{0}=q$ and $G_{0}=G$ and $N_{0}=N^{\prime}$ and $\left\langle\beta_{l}: l \in \omega\right\rangle$ extends $\left\langle\beta_{l}: l<k\right\rangle$, and for every $n \in \omega$ we have that each of the following holds:

(1) $p_{n} \|^{\text {" }} G_{n}^{\prime}=G_{n} \uparrow \alpha_{n+1}$ and $G_{n+1}=G_{n} / G_{n}^{\prime}$ (see the successor case, above)," and

(2) $p_{n} \Vdash " N_{n+1}$ is a countable elementary substructure of $H_{\chi}\left[G_{P_{\alpha_{n}}}\right]$ and $\left\{N_{n}\left[G_{P_{\alpha_{n-1}, \alpha_{n}}}\right], G_{n}, f_{n}, \alpha_{n+1}, \sigma_{n}\right\} \in N_{n+1} \in N\left[G_{P_{\alpha_{n}}}\right]$ " (if $n=0$ then replace $N_{0}\left[G_{P_{\alpha_{-1}, \alpha_{0}}}\right]$ with $\left.N_{0}\right)$, and

(3) $\beta_{n}$ is a $P_{\alpha_{n}}$-name for an integer and $p_{n} \Vdash$ " $\left(\forall j \geq \beta_{n}\right)(g$ does not extend $\left.f_{n}\left[G_{n}\right](j)\right)$," and

(4) $p_{n} \Vdash$ " $G_{n}^{*} \subseteq P_{\alpha_{n}, \alpha_{n+1}} \cap N_{n+1}$ is $N_{n+1}$-generic and $q_{n} \rrbracket \alpha_{n+1} \in G_{n+1}^{*} \in$ $N\left[G_{P_{\alpha_{n}}}\right]$ and $(\forall l<\max (n+1, k))\left(\forall j \geq \beta_{l}\right)\left(g\right.$ does not extend $\left.f_{l}\left[G_{n+1}\right]\left[G_{n}^{*}\right](j)\right)$," and

(5) $p_{n+1} \in P_{\alpha_{n+1}}$ is $N$-generic and $p_{n+1} \Vdash$ " $g$ is random over $N\left[G_{P_{\alpha_{n+1}}}\right]$ and $(\forall i<\max (n+1, k))\left(\forall j \geq \beta_{l}\right)\left(g\right.$ does not extend $\left.f_{l}\left[G_{n+1}\right](j)\right)$," and

(6) $p_{n} \Vdash$ " $p_{n+1} \uparrow\left[\alpha_{n}, \alpha_{n+1}\right) \leq q_{n} \uparrow \alpha_{n+1}$," and

(7) $p_{n+1} \Vdash " q_{n+1} \leq q_{n} \uparrow\left[\alpha_{n+1}, \kappa\right)$ and $q_{n+1} \in G_{n+1}$ and $q_{n+1}$ decides the value of $\sigma_{n}$ and $q_{n+1}$ decides the value of $f_{l} \uparrow n$ for every $l \leq n$."

The construction proceeds as follows. Given $p_{n}$ and $q_{n}$ and $G_{n}$, construct $G_{n}^{\prime}$ and $G_{n+1}$ as in (1) (see successor case, above). There is no problem in choosing $N_{n+1}$ as in (2). We have that $p_{n} \Vdash$ " $f_{n}\left[G_{n}\right] \in \mathcal{B}^{\prime}$ " by the reasoning of Claim 1 in the proof of Lemma 6.5 , hence we may choose $\beta_{n}$ as in (3) because of Lemma 6.2. We may choose $G_{n}^{*}$ as in (4) by Lemma 6.5 . We may choose $p_{n+1}$ satisfying (5) and (6) by using the induction hypothesis. There is no difficulty in choosing $q_{n+1}$ satisfying $(7)$.

Take $r \in P_{\kappa}$ such that for every $n \in \omega$ we have $r \uparrow \alpha_{n}=p_{n}$.

Claim. $r \|$ " $g$ is random over $N\left[G_{P_{\kappa}}\right]$.

Proof: Suppose not. By Lemma 6.2 we may take $r^{\prime} \leq r$ and $l \in \omega$ such that $r^{\prime} \Vdash \models^{\prime}\left(\exists^{\infty} m \in \omega\right)\left(g\right.$ extends $\left.f_{l}(m)\right)$." By strengthening $r^{\prime}$ further, we may assume there is an integer $\beta^{*}$ such that $r^{\prime} \Vdash$ " $\beta_{l}=\beta^{*}$." By a further strengthening of $r^{\prime}$ 
we may assume there is an integer $j \geq \beta^{*}$ such that $r^{\prime} \Vdash$ " $g$ extends $f_{l}(j)$." Let $n=\max (j+1, l+1)$. By $(7)$ we have that $p_{n+1} \Vdash$ " $q_{n+1} \Vdash{ }^{\prime} f_{l}\left[G_{n+1}\right](j)=f_{l}(j)$. ." We have $p_{n+1} \Vdash$ " $g$ does not extend $f_{l}\left[G_{n+1}\right](j)$." The Claim is established.

We have that $r$ is $N$-generic by the usual argument on ordinal names in $N$, and it is clear that $r \|$ " $(\forall l<k)\left(\forall j \geq \beta_{l}\right)\left(g\right.$ does not extend $\left.f_{l}(j)\right)$."

The Theorem is established.

The following Theorem is [9, Claim XVIII.3.8C(1)].

Theorem 6.9. Suppose $\left\langle P_{\eta}: \eta \leq \kappa\right\rangle$ is a countable support iteration based on $\left\langle Q_{\eta}: \eta<\kappa\right\rangle$ and for every $\eta<\kappa$ we have $\mathbf{1} \Vdash_{P_{\eta}}$ " $Q_{\eta}$ is proper and for every

$g \in{ }^{\omega} 2$ we have that $Q_{\eta}$ is $g$-good." Then $V\left[G_{P_{\kappa}}\right] \models " \omega 2 \cap V$ does not have measure zero."

Proof: By Theorem 6.8 with $\alpha=k=0$ and Lemma 6.2.

\section{Preservation of "the set of old reals is non- meager"}

Let $\mathcal{B}^{*}$ be the set of functions from ${ }^{<\omega} 2$ into ${ }^{<\omega} 2$.

Definition 7.1. Suppose $f \in \mathcal{B}^{*}$ and $g \in{ }^{\omega} 2$. We say $f R^{\dagger} g$ iff $\left(\exists^{\infty} m \in \omega\right)$ $\left(g\left\lceil m^{\wedge} f(g \uparrow m)\right.\right.$ is an initial segment of $\left.g\right)$.

Lemma 7.2. Suppose $X \subseteq{ }^{\omega} 2$. Then $X$ is non-meager iff for every $f \in \mathcal{B}^{*}$ there is $g \in X$ such that $f R^{\dagger} g$.

Proof: Suppose $X$ is non-meager, and suppose $f \in \mathcal{B}^{\prime}$.

For every $i \in \omega$ let $D_{i}=\bigcup\left\{U_{\tau^{\wedge} f(\tau)}:(\exists n>i)\left(\tau \in{ }^{n} 2\right)\right\}$. We have that each $D_{i}$ is an open dense set, so because $X$ is non-meager, we may fix $g \in X \cap \bigcap\left\{D_{i}\right.$ : $i \in \omega\}$. Clearly $f R^{\dagger} g$.

For the converse, suppose $\left(\forall f \in \mathcal{B}^{*}\right)(\exists g \in X)\left(f R^{\dagger} g\right)$, and suppose $\left\langle D_{i}: i \in \omega\right\rangle$ is a decreasing sequence of open dense subsets of ${ }^{\omega} 2$. We show $X \cap \bigcap\left\{D_{i}: i \in \omega\right\}$ is non-empty. It suffices to find $g \in X$ such that $\left(\exists^{\infty} j \in \omega\right)\left(g \in D_{j}\right)$.

Choose $f \in \mathcal{B}^{*}$ such that for every $\eta \in<\omega_{2}$ we have $U_{\eta^{\wedge} f(\eta)} \subseteq D_{\operatorname{lh}(\eta)}$. Fix $g \in X$ such that $f R^{\dagger} g$. Given $i \in \omega$ choose $j>i$ such that $g g^{\uparrow} j^{\wedge} f\left(g^{\uparrow} j\right)$ is an initial segment of $g$. Let $\eta=g \uparrow j$. Then $g \in U_{\eta^{\wedge} f(\eta)} \subseteq D_{j}$.

The Lemma is established. 
Lemma 7.3. Suppose $\lambda$ is a sufficiently large regular cardinal and $N$ is a countable elementary substructure of $H_{\lambda}$, and suppose $g \in{ }^{\omega} 2$. The following are equivalent:

(1) $\left(\forall f \in \mathcal{B}^{*} \cap N\right)\left(f R^{\dagger} g\right)$.

(2) $\left(\forall f \in \mathcal{B}^{*} \cap N\right)(\exists m \in \omega)\left(g\left\lceil m^{\wedge} f(g\lceil m)\right.\right.$ is an initial segment of $g)$.

(3) $g$ is Cohen over $N$.

Proof: It is obvious that (1) implies (2).

Suppose (2) holds and $D \in N$ is an open dense subset of $\omega_{2}$. Choose $f \in$ $\mathcal{B}^{*} \cap N$ such that $\left(\forall \nu \in{ }^{<\omega} 2\right)\left(U_{\nu^{\wedge} f(\nu)} \subseteq D\right)$. Using (2), choose $m \in \omega$ such that $g\left\lceil{ }^{\wedge} f\left(g\lceil m)\right.\right.$ is an initial segment of $g$. We have $g \in U_{g \uparrow^{\wedge} f(g \uparrow m)} \subseteq D$. We conclude that $g \in \bigcap\left\{D \in N: D\right.$ is an open dense subset of $\left.{ }^{\omega} 2\right\}$, i.e., $g$ is Cohen over $N$.

Finally, suppose (3) holds and $f \in \mathcal{B}^{*} \cap N$. Suppose $k \in \omega$. Let $D_{k}=\left\{h \in{ }^{\omega} 2\right.$ : $(\exists m>k)\left(h\left\lceil m^{\wedge} f(h\lceil m)\right.\right.$ is an initial segment of $\left.h)\right\}$. It is easy to see that for every $k \in \omega$ we have $D_{k}$ is an open dense subset of ${ }^{\omega} 2$. Because $(\forall k \in \omega)\left(g \in D_{k}\right)$ we have that $f R^{\dagger} g$.

The Lemma is established.

The following Lemma, due to Goldstern and Shelah, is [9, Lemma XVIII.3.11].

Lemma 7.4. Suppose $P$ is a Suslin proper forcing (see [1, Section 7]) and for every forcing $Q$ we have $\mathbf{1} \Vdash_{Q}$ " $P$ is Suslin proper and $\mathbf{1} \|_{P}{ }^{\text {c } \omega_{2}} \cap V\left[G_{Q}\right]$ is not meager.'" Suppose $\lambda$ is a sufficiently large regular cardinal and $N$ is a countable elementary submodel of $H_{\lambda}$ and $P \in N$ and $p \in P \cap N$ and $g \in{ }^{\omega_{2}}$ is Cohen over $N$. Then there is $q \leq p$ such that $q$ is $N$-generic and $q \Vdash$ " $g$ is Cohen over $N\left[G_{P}\right] . "$

The proof presented in [9] is quite clear, so we do not repeat it here.

Lemma 7.5. Suppose $\left\langle P_{\eta}: \eta \leq \kappa\right\rangle$ is a countable support forcing iteration based on $\left\langle Q_{\eta}: \eta<\kappa\right\rangle$. Suppose for every $\eta<\kappa$ we have $\mathbf{1} \|_{P_{\eta}}$ " $Q_{\eta}$ is a Suslin proper forcing and for every forcing $Q$ we have $\mathbf{1} \|_{Q}$ ' $Q_{\eta}$ is Suslin proper and $\mathbf{1} \|_{Q_{\eta}}$ "w $2 \cap V\left[G_{P_{\eta}}\right]\left[G_{Q}\right]$ is not meager." " Suppose $\lambda$ is a sufficiently large regular cardinal and $N$ is a countable elementary substructure of $H_{\lambda}$ and $P_{\kappa} \in N$ and $\alpha \in \kappa \cap N$ and $p \in P_{\alpha}$ is $N$-generic and $p \|$ " $q \in P_{\alpha, \kappa} \cap N\left[G_{P_{\alpha}}\right]$ and $g \in \omega_{2}$ is Cohen over $N\left[G_{P_{\alpha}}\right]$." Then there is $r \in P_{\kappa}$ such that $r$ is $N$-generic and $r \uparrow \alpha=p$ and $p \|$ " $r \uparrow[\alpha, \kappa) \leq q$ " and $r \|$ " $g$ is Cohen over $N\left[G_{P_{\kappa}}\right]$."

Proof: By induction on $\kappa$. 
Case 1: $\kappa$ is a successor ordinal.

Let $\beta$ be the immediate predecessor of $\kappa$. By the induction hypothesis we may take $r^{\prime} \in P_{\beta}$ such that $r^{\prime}$ is $N$-generic and $r^{\prime} \uparrow \alpha=p$ and $p \|-{ }^{\prime} r^{\prime} \leq q \uparrow \beta "$ and $r^{\prime} \Vdash$ " $g$ is Cohen over $N\left[G_{P_{\beta}}\right]$." By Lemma 7.4 we may take $r^{*} \in Q_{\beta}$ such that $r^{\prime} \|{ }^{\prime} r^{*} \leq q(\beta)$ and $r^{*}$ is $N\left[G_{P_{\beta}}\right]$-generic and $r^{*} \| \vdash^{\prime} g$ is Cohen over $N\left[G_{P_{\beta}}\right]\left[Q_{\beta}\right]$.'" Let $r \in P_{\kappa}$ be defined by $r \beta=r^{\prime}$ and $r(\beta)=r^{*}$. We have that $r$ satisfies the requirements of the Lemma.

Case 2: $\kappa$ is a limit ordinal.

Let $\left\langle\alpha_{k}: k \in \omega\right\rangle$ be an increasing sequence from $\kappa \cap N$ cofinal in $\sup (\kappa \cap N)$ such that $\alpha_{0}=\alpha$. Let $\left\langle\sigma_{k}: k \in \omega\right\rangle$ list all $P_{\kappa}$-names $\sigma$ in $N$ such that $\mathbf{1} \|_{P_{\kappa}}$ " $\sigma$ is an ordinal."

Let $\left\langle f_{i}: i \in \omega\right\rangle$ list all $P_{\kappa}$-names $f$ in $N$ such that $V\left[G_{P_{\kappa}}\right] \models " f \in \mathcal{B}^{*}$," and let $\left\langle\eta_{m}^{\prime}: m \in \omega\right\rangle$ list $<\omega_{2}$.

Build $\left\langle q_{k}, p_{k}, n_{k}: k \in \omega\right\rangle$ such that $p_{0}=p$ and $q_{0}=q$ and for every $k \in \omega$ we have that each of the following holds:

(1) $p_{k} \in P_{\alpha_{k}}$ is $N$-generic, and

(2) $p_{k} \Vdash " q_{k+1} \in P_{\alpha_{k}, \kappa} \cap N\left[G_{P_{\alpha_{k}}}\right]$ and $q_{k+1} \leq q_{k} \uparrow\left[\alpha_{k}, \kappa\right)$," and

(3) $p_{k} \Vdash{ }^{\prime} " q_{k+1} \Vdash{ }^{\prime} g$ is Cohen over $N\left[G_{P_{\alpha_{k}}}\right]$ and $\sigma_{k} \in N$ and $n_{k} \in \omega$ and $g\left\lceil n_{k} \hat{f_{k}}\left(g \uparrow n_{k}\right)\right.$ is an initial segment of $g, '$ ' and

(4) $p_{k+1} \uparrow \alpha_{k}=p_{k}$, and

(5) $p_{k} \Vdash " p_{k+1} \uparrow\left[\alpha_{k}, \alpha_{k+1}\right) \leq q_{k+1} \uparrow \alpha_{k+1}$."

The construction proceeds as follows. Given $p_{k}$ and $q_{k}$, work in $V\left[G_{P_{\alpha_{k}}}\right]$ with $p_{k} \in G_{P_{\alpha_{k}}}$.

Build $\left\langle q_{k}^{m}: m \in \omega\right\rangle \in N\left[G_{P_{\alpha_{k}}}\right]$ and $f_{k}^{\prime} \in \mathcal{B}^{*} \cap N\left[G_{P_{\alpha_{k}}}\right]$ such that $\left\langle q_{k}^{m}: m \in \omega\right\rangle$ is a decreasing sequence of elements of $P_{\alpha_{k}, \kappa}$ and $q_{k}^{0} \leq q_{k} \uparrow\left[\alpha_{k}, \kappa\right)$ and there is an ordinal $\tau$ such that $q_{k}^{0} \Vdash$ " $\tau=\sigma_{k}$," and for every $m \in \omega$ we have that $q_{k}^{m} \Vdash " f_{k}^{\prime}\left(\eta_{m}^{\prime}\right)=f_{k}\left(\eta_{m}^{\prime}\right)$." Necessarily $\tau \in N\left[G_{P_{\alpha_{k}}}\right]$ and therefore, because $p_{k} \in G_{P_{\alpha_{k}}}$ is $N$-generic, we have $\tau \in N$. Because $g$ is Cohen over $N\left[G_{P_{\alpha_{k}}}\right]$ we may use Lemma 7.3 to take $n_{k}$ such that $g \uparrow n_{k} f_{k}^{\prime}\left(g\left\lceil n_{k}\right)\right.$ is an initial segment of g.

Let $q_{k+1}=q_{k}^{n_{k}+1}$.

Using the induction hypothesis, we may choose $p_{k+1}$ as required.

This completes the recursive construction.

Let $r \in P_{\kappa}$ be such that for every $k \in \omega$ we have $r \uparrow \alpha_{k}=p_{k}$. 
We have that $r$ is $N$-generic, because for each $k \in \omega$ we have $p_{k+1} \Vdash$ " $q_{k+1} \Vdash$ ' $\sigma_{k} \in N$.'”

Suppose, towards a contradiction, that $r^{\prime} \leq r$ and $r^{\prime} \|$ " $g$ is not Cohen over $N\left[G_{P_{\kappa}}\right] . "$ Choose $r^{*} \leq r^{\prime}$ and $k \in \omega$ such that $r \Vdash "(\forall m \in \omega)\left(g \uparrow m^{\wedge} f_{k}(g\lceil m)\right.$ is not an initial segment of $g$ )."

Because $r^{*} \leq\left(p_{k+1}, q_{k+1}\right)$ we have $r^{*} \Vdash{ }^{\prime} g_{n_{k}}{ }^{\wedge} f_{k}\left(g\left\lceil n_{k}\right)\right.$ is an initial segment of g." This is a contradiction.

The Lemma is established.

The following Theorem is [9, Claim XVIII.Claim 3.10C].

Theorem 7.6. Suppose $\left\langle P_{\eta}: \eta \leq \kappa\right\rangle$ is a countable support forcing iteration based on $\left\langle Q_{\eta}: \eta<\kappa\right\rangle$. Suppose for every $\eta<\kappa$ we have $\mathbf{1} \Vdash_{P_{\eta}}$ " $Q_{\eta}$ is Suslin proper forcing and for every forcing $Q$ we have $\mathbf{1} \|_{Q}$ ' $Q_{\eta}$ is Suslin proper and $\mathbf{1} \Vdash_{Q_{\eta}}{ } \omega_{2} \cap V\left[G_{P_{\eta}}\right]\left[G_{Q}\right]$ is not meager." " Then $\mathbf{1} \Vdash_{P_{\kappa}}{ } " \omega 2 \cap V$ is not meager."

Proof: Suppose, towards a contradiction, that $q \in P_{\kappa}$ and $q \|^{" \omega \omega} 2 \cap V$ is meager." By Lemma 7.3 we may take $f$ a $P_{\kappa}$-name for an element of $\mathcal{B}^{*}$ such that $q \|$ " $\left(\forall g \in{ }^{\omega} 2 \cap V\right)\left(f R^{\dagger} g\right.$ fails $)$." Let $\lambda$ be a sufficiently large regular cardinal and let $N$ be a countable elementary substructure of $H_{\lambda}$ such that $\left\{P_{\kappa}, q, f\right\} \in N$.

Let $g \in{ }^{\omega} 2$ be Cohen over $N$.

By Lemma 7.5 with $\alpha=0$ we may take $r \leq q$ such that $r \Vdash$ " $g$ is Cohen over $N\left[G_{P_{\kappa}}\right]$." By Lemma 7.3 we have $r \Vdash$ " $f R^{\dagger} g$." This contradiction establishes the Theorem.

\section{References}

[1] Bartoszynski, T., and H. Judah, Set Theory: On the Structure of the Real Line, A K Peters, 1995.

[2] Goldstern, M., Tools for Your Forcing Construction, Set theory of the reals (Haim Judah, editor), Israel Mathematical Conference Proceedings, vol. 6, American Mathematical Society, pp. 305-360. (1993)

[3] Goldstern, M., and J. Kellner, New reals: Can live with them, can live without them, Math. Log. Quart. 52, No. 2, pp. 115-124, 2006.

[4] Kellner, J., and S. Shelah, Preserving preservation, Journal of Symbolic Logic, vol. 70, pp. 914-945, 2005. 
[5] Moschovakis, Y., Descriptive Set Theory, Studies in Logic and the Foundations of Mathematics, v. 100, North-Holland, 1980.

[6] Schlindwein, C., Shelah's work on non-semi-proper iterations, I, Archive for Mathematical Logic, vol. 47, pp. 579-606, 2008.

[7] Schlindwein, C., Shelah's work on non-semi-proper iterations, II, Journal of Symbolic Logic, vol. 66, pp. 1865-1883, 2001.

[8] Schlindwein, C., Understanding preservation theorems: Chapter VI of Proper and Improper Forcing," submitted.

[9] Shelah, S., Proper and Improper Forcing, Perspectives in Mathematical Logic, Springer, Berlin, 1998. 